\title{
Seasonal ataxia: a case report of a disappearing disease
}

Adebiyi Ayoade Moyo ${ }^{1 *}$,Fawale Michael Bimbo ${ }^{1}$, Komolafe Morenikeji Adeyoyin ${ }^{1}$, Amadi Valentine Nnaemeka ${ }^{1}$, Ganiyu Oluwatoyin $^{1}$, Adeyeye Victor Oladeji ${ }^{1}$

1 Department of Medicine, Obafemi Awolowo University, Ile-Ife, Nigeria.

Adebiyi Ayoade Moyo :ayoademoyo@gmail.com

Fawale Michael Bimbo: bimbofawale@yahoo.com

Komolafe Morenikeji Adeyoyin: adeyoyin2001@yahoo.com

Amadi Valentine Nnaemeka:mekuslala@yahoo.com

Ganiyu Oluwatoyin: abidemi@yahoo.com

Adeyeye Victor Oladeji: vioyeye@yahoo.com

\begin{abstract}
:
Introduction: Seasonal ataxia is a clinical syndrome of acute cerebellar ataxia which follows ingestion of roasted larvae of Anaphe venata Butler, an alternative protein source consumed in western Nigeria. It was first reported in the 1950s in western Nigeria when it caused a wave of epidemics. This is the first case report of this condition in the literature since 1993.

Case report: We present the case of a 35 year old woman from western Nigeria who was admitted in October 2012 with acute onset of gait instability and bilateral hand tremors, preceded by several episodes of vomiting. She had ingested a meal containing roasted larvae of the African silkworm, 2 hours before the onset of vomiting.

Conclusion: Seasonal ataxia is an important differential diagnosis of acute cerebellar ataxia among the indigenous ethnic population of western Nigeria.It is non-fatal and treatable, with complete resolution of symptoms usually following thiamine therapy.
\end{abstract}

Keywords: Anaphe venata, Nigeria, ataxia, seasonal.

DOI: http://dx.doi.org/10.4314/ahs.v14i3.38

\section{Introduction}

Outbreaks of a peculiar syndrome of acute cerebellar ataxia have occurred in parts of Southwestern Nigeria since the 1950s, especially coinciding with the rainy reason. The earliest report of this condition, which was initially identified as Encephalitis tremens, was made by Wright and Morley in 1958 [1]. Their observations were based on the clinical presentations of patients of Ijesha ethnic stock who presented with an acute flu-like illness associated with tremors. Epidemics have since occurred in several parts of western Nigeria as documented by other workers [1]. Osuntokun investigated 50 patients involved in the 1972 epidemic [2] and noted that symptoms commenced usually within three hours of the local staple meal of yam, with the earliest symptom being vomiting. In his report, the most frequent symptom found was a 'parkinsonian' tremor with coexisting cerebellar signs. This syndrome was thought to be due to cholinergic hyperstimulation resulting from a substance in yam which probably depleted endogenous acetylcholinesterase. Subsequent work by Adamolekun and hiscol laborators has elucidated the aetiopathoge- netic basis of this peculiarly Nigerian ataxic syndrome as well as providing comprehensive clinico-epidemiological profiles of the disease. It was noted that the disorder occurred more frequently in western Nigeria, usually following ingestion of roasted larvae of Anaphe venata butler [3], [4]. Anaphe is a defoliator of certain timber species-triplochyton scleroxylon- indigenous to the tropical rainforest of western Nigeria. These larvae are consumed in combination with carbohydrate based foods during the rainy season from July- October when they are amply available in local markets. They have been shown to be rich in amino acids [5]. It has however been reported that the larvae of anaphe venata contain heat-resistant thiaminases, [6], [7] which upon ingestion further deplete the already low thiamine levels in the at-risk population and thereby creates optimal conditions for seasonal ataxia to occur. It has been noted that low serum albumin levels [8] act as a potentiating factor to trigger the clinical consequences of acute thiamine deficiency. More recently it has been suggested that the cerebellar symptoms of seasonal ataxia may be mediated by increased GABAergic, opioid and cholinergic

\footnotetext{
Corresponding author

( Adebiyi Ayoade Moyo)

Department of Medicine, Obafemi Awolowo

University, Ile-Ife, Nigeria.

Adebiyi Ayoade Moyo :ayoademoyo@gmail.com
} 
neurotransmission [9].

A sex predilection has been reported in the occurrence of seasonal ataxia, with a female to male ratio of 3.25:1 and all age groups are vulnerable(2). The disease typically runs a benign self-limited course $(2,3$.) The most common presenting symptoms in patients with this disorder are tremors, unsteadiness while walking or standing, vomiting and dizziness (2,3). Less frequent symptoms include confusion, palpitations, excessive salivation, diarrheoa and urinary retention. Neurological findings include cerebellar ataxia and intention tremors which are found in virtually all patients (2), as well as dysarthria, nystagmus, cogwheel rigidity and impaired consciousness. Diagnosis is chiefly clinical but investigations may be conducted to exclude differential diagnoses and complications. The clinical features of seasonal ataxia are often completely reversed by administration of high dose thiamine infusions [10]. Antibiotics have no role in the treatment of this condition [11]. Recent work in animals suggests that anticholinergics and opiate antagonists may help to reverse the symptoms of seasonal ataxia (9). Public health interventions in affected communities in the 1990s, discouraging anaphe consumption appear to have brought about a remarkable decline in occurence of this disease (11).

\section{Case presentation}

The patient is a 35 year old seamstress; a widowed mother of four children who was brought to the emergency department with persistent vomiting of 12 hours. Vomiting started 2 hours after ingestion of a meal containing roasted larvae of African silkworm, procured from the local market. Two of her children had shared in the meal with her but had no symptoms. Before presentation she had a total of fifteen episodes of vomiting with ensuing fatigue and dizziness. There was moderate abdominal discomfort but no history of diarrheoa. There was no alteration in consciousness and no other remarkable symptoms were reported at presentation. She had not ingested alcohol or any other drugs prior to onset of symptoms and she did not report a history of amenorrheoa.A few hours after admission, she developed bilateral hand tremors with gait unsteadiness. Slurring of speech and visual disturbances were not reported. She had never been previously diagnosed with hypertension and she had no past history of stroke. There was no past history of similar illness despite habitual ingestion of the larvae in past rainy seasons.

Initial neurologic examination in the emergency department was normal. Admission blood pressure was $120 / 80 \mathrm{mmHg}$, she was noted to be dehydrated but was afebrile. A repeat neurologic examination 24 hours af- ter admission revealed a conscious patient with blepharoclonus, bilateral horizontal jerk nystagmus, intention tremors in the upper limbs, right upper limb cogwheel rigidity, truncal and gait ataxia. Muscle power was grade 5 ( Medical Research Council grading) in all muscle groups, with decreased deep tendon reflexes and bilateral flexorplantar response. Romberg's sign was negative and all sensory modalities were normal.

Serum urea, electrolytes and creatinine were normal with urea $2.2 \mathrm{mmol} / \mathrm{L}$, sodium $134 \mathrm{mmol} / \mathrm{L}$, potassium $4.4 \mathrm{mmol} / \mathrm{L}$, bicarbonate $24 \mathrm{mmol} / \mathrm{L}$ and creatinine 96 umol/L. There were no abnormalities on the complete blood count, with packed cell volume of $40 \%$, total white cell count of $8,000 /$ cubic mm, differential count 65\% neutrophils, 25\% lymphocytes,eosinophils 5\% and basophils 5\%. Her haematocrit was $13.5 \mathrm{~g} / \mathrm{dL}$. Serum albumin was $35 \mathrm{~g} / \mathrm{L}$ which is low normal and total protein was $72 \mathrm{~g} / \mathrm{L}$. She could not afford neuroimaging ( cranial magnetic resonance imaging). Pregnancy test using urine test strip was negative and retroviral screen with a rapid test kit was also negative. Admission random plasma glucose was normal with a value of 7.1 $\mathrm{mmol} / \mathrm{L}$.

She received 4 litres of intravenous isotonic saline and ringers lactate infusions in the first 24 hours following admission.Parenteral and oral thiamine were not available, hence 10 cubic centimeters of injectable B complex vitamins was added to each pint of infusion fluid and she was also given oral B complex vitamins. She was also given parenteral anti-emetics and antibiotics. Her symptoms resolved completely within 72 hours of presentation.

At follow-up in the clinic two weeks after discharge she had no symptoms or signs of cerebellar dysfunction. She was further seen after six weeks and was discharged from the clinic on the grounds of sustained clinical improvement.

\section{Discussion}

The temporal course of the case presented fits the clinical description documentedby Osuntokun (3): the earliest symptom in this patient was vomiting, preceding other symptoms by a 12 hour duration, and developing 2 hours after the meal. The prominence of vomiting following a meal, in the absence of diarrheoa and other gastrointestinal symptoms, initially led the admitting physicians to diagnose food poisoning. It was only with further evolution of the symptomatology that the clinical picture of acute ataxic syndrome emerged.

Acute cerebellar ataxias in adults may result from the alcohol-induced Wernicke encephalopathy, or from ingestion of anticonvulsants like phenytoin, but these 
factors were considered unlikely in this patient as there had been no prior exposure to these agents. Cerebellar haemorrhage or infarct are also possible differentials however the absence of stroke risk factors such as hypertension, coupled with the rapid symptom resolution and the occurence of persistent vomiting after a meal suggest an alternative diagnosis. Infections of the cerebellum such as abscess or encephalitis were ruled out by the absence of fever or altered consciousness as well as the normal white cell count.

In the light of findings by previous workers $(3,9)$, it is not unusual that the subject was the only participant in the culprit meal who developed symptoms of the disease. One worker has documented that only one of every four participants in a predisposing meal will develop symptoms (3). This pattern has been attributed to low levels of serum albumin in individuals who eventually develop the disease after exposure to the aetiologic agent (9). The clinico-epidemiologic descriptors of the subject namely female gender, low socioeconomic status, occurrence in October (rainy season) and the sudden onset of transient cerebellar ataxia, are all consistent with previous documentation of the disease $(2,11)$. The absence of other features of increased cholinergic activity such as excessive salivation, diarrheoa and urinary retention in this patient is not out of place as few patients in past series had these symptoms $(2,3)$. The results of baseline investigations of the subject were normal but this does not contradict the diagnosis since supportive investigation results are not required to make a definitive diagnosis of this disease. The index case satisfied the diagnostic criteria developed by Adamolekun, namely: acute cerebellar ataxia following a meal of anaphe venata, with or without nystagmus and impaired consciousness, occurring between July and October (2). The dramatic resolution of symptoms probably in response to vitamin supplementation is not unexpected in the light of past work that has demonstrated the therapeutic efficacy of thiamine in treating this seasonal ataxic syndrome (10). In a previous report of seasonal ataxia in a pregnant Nigerian woman, the time from presentation to resolution of symptoms was 48 hours [12]

\section{Conclusion}

Seasonal ataxia should be considered in the differential diagnosis of acute cerebellar ataxia in the indigenous people of western Nigeria. Epidemics of seasonal ataxic syndrome may occur if economic conditions worsen and people ingest culturally acceptable alternative protein sources such as Anaphe venata. Public awareness of seasonal ataxia must be sustained in the relevant communities in order to prevent occurrence of the disease.

\section{Conflict of interest}

The authors declare that they have no potential or actual conflicts of interest.

\section{References}

1. Wright J,Morley DC .Encephalitis tremens. Lancet 1958, 1:871-873.

2. Adamolekun B, Ibikunle F R.Investigation of an epidemic of seasonal ataxia in Ikare, western Nigeria. Acta Neurol Scand 1994, 90(5):309-311

3. Osuntokun B O.Epidemic ataxia in Western Nigeria. Br Med J 1972, 2:589.

4. Adamolekun B.A seasonal ataxic syndrome in Southwestern Nigeria: an aetiological hypothesis of acute thiamine deficiency. Ethn Dis 1992,2(2):185-186

5. Adamolekun B. Anaphe venata entomophagy and seasonal ataxic syndrome in southwest Nigeria. Lancet 1993, 341(8845):629

6. Ashiru M O. The food value of the larvae of anaphe venata butler. Ecology of food and nutrition 1998, 28:227-242.

7. Nishimune T, Watanabe Y, Okazaki H, Akai H. Thiamine is decomposed due to anaphe spp. entomophagy in seasonal ataxia patients in Nigeria. J Nutr 2000, 130(6):1625-1628.

8. Okonji RE, Bamitale KDS, Balogun RO. Biochemical properties of thiaminase from anaphe venata Butler. African Journal of Biotechnology 2012, 11(20):45434550.

9. Adamolekun B, Lawal T, Ndububa D A, Balogun M O. Serum albumin levels and intraethnic variations in susceptibility to a seasonal ataxia in Nigerians. Ethn Dis 1994, 4(1): 87-90.

10. Bamitale K D S, Akanmu M A, Ukponmwan O E. Neuropharmacological profile of aqueous extract of anaphe venata larva (notodontidae) in rats. Afr J Tradit Complement Altern Med 2011, 8(3):260-266.

11. Adamolekun B, Adamolekun WE, Sonibare AD, Sofowora G. A double blind, placebo-controlled study of the efficacy of thiamine hydrochloride in a seasonal ataxia in Nigerians. Neurology 1994, 44(3):549-551.

12. Adamolekun B.Seasonal ataxia of western Nigeria: Evaluation of impact of health education on hospital prevalence. J Epidemiol Community Health 1995, 49(5):489-491.

13 Adamolekun B, Faleyimu BL, Selo-Ojeme D. Seasonal ataxic syndrome in a pregnant Nigerian woman. Intl J Gyn Obs 1993, 41(2):187-188. 